\title{
Comprometimento cognitivo e funcional em pacientes acometidos de acidente vascular encefálico: Importância da avaliaçáo cognitiva para intervenção na Terapia Ocupacional
}

Andressa de Oliveira Ferro ${ }^{\mathrm{a}}$, Ana Elizabeth dos Santos Lins ${ }^{\mathrm{b}}$, Euclides Maurício Trindade Filho ${ }^{\mathrm{c}}$

aTerapeuta ocupacional. Graduada pela Universidade Estadual de Ciências da Saúde

de Alagoas - UNCISAL, Maceió, AL, Brasil. Pós-graduanda em Reabilitação Neurofuncional,

Centro de Estudos Superior de Maceió - CESMAC, Maceió, AL, Brasil

${ }^{\text {b}}$ Terapeuta ocupacional, professora do Curso de Terapia Ocupacional da Universidade Estadual de Ciências da Saúde de Alagoas - UNCISAL, Maceió, AL, Brasil. Gerontóloga, Sociedade Brasileira de Geriatria e

Gerontologia. Especialista em Saúde do Idoso e em Saúde Pública. Mestre em Ciências.

Coordenadora geral do Programa de Extensão Interdisciplinar Pró-Idoso e professora da Universidade Aberta à Terceira Idade - UATI, São Paulo, SP, Brasil

'Médico graduado, Universidade Federal de Alagoas - UFAL, Especialização em Saúde Pública, Fundação Oswaldo Cruz - Fiocruz. Especialização em Neurofisiologia Clínica, Universidade Federal de São Paulo - UNIFESP, Mestrado em Ciências Biológicas (Fisiologia), Universidade Federal de

Pernambuco - UFPE, Doutorado em Neurociências, Universidade Federal de São Paulo - UNIFESP, Pós-doutorado pela Universidade Federal de São Paulo - UNIFESP, Atualmente é funcionário da Universidade Estadual de Ciências da Saúde de Alagoas - UNCISAL, Maceió, AL, Brasil, professor adjunto da Faculdade de Alagoas, professor da Fundação Educacional Jaime de Altavila, Maceió, AL, Brasil

Resumo: Introdução: Acidentes vasculares encefálicos (AVE) podem gerar déficits motor, sensorial e cognitivo, repercutindo no desempenho do indivíduo nas atividades cotidianas. Alteração em qualquer área cognitiva afeta o engajamento ocupacional do indivíduo. Objetivo: Avaliar a capacidade cognitiva e funcional em pacientes acometidos de AVE, mostrando a importância da avaliação cognitiva para a intervenção do terapeuta ocupacional. Método: Estudo comparativo de delineamento transversal com amostra de 44 indivíduos entre 30 e 80 anos, de ambos os sexos. Os sujeitos foram distribuídos em três grupos: grupo Adulto: 11 indivíduos acometidos por AVE, com idade entre 30 e 59 anos; grupo Idoso: 10 indivíduos acometidos por AVE, com idade entre 60 e 80 anos; grupo Controle: 23 indivíduos normais, com idade entre 30 e 80 anos. Testes avaliados: MEEM, teste do relógio, teste das trilhas A e B e da capacidade funcional (BOMFAQ). Resultados: Alterações cognitivas foram identificadas nos grupos Adulto e Idoso. O grupo Adulto mostrou pior desempenho no teste do relógio quando comparado ao grupo Controle. Os grupos Adulto e Idoso foram piores no desempenho do teste trilha A (atenção). Ao utilizarem-se números absolutos do teste trilha B (atenção visual, habilidade grafomotora e flexibilidade mental), não houve diferença significativa nos grupos Adulto e Idoso em relação ao grupo Controle. O grupo Adulto apresentou maior prevalência de comprometimento moderado/grave na realização das atividades cotidianas. Conclusão: Indivíduos acometidos de AVE, na grande maioria, além de apresentarem comprometimento da capacidade funcional apresentam alterações cognitivas que repercutem de modo negativo na realização das tarefas cotidianas, sejam elas ocupacionais, de lazer ou de autocuidado. Verificou-se a necessidade da avaliação cognitiva para melhor direcionamento da reabilitação e melhora na qualidade de vida.

Palavras-chave: Acidente Vascular Encefálico, Cognição, Funcionalidade, Terapia Ocupacional. 


\title{
Cognitive and functional impairment in patients suffering from stroke: the importance of cognitive assessment for Occupational Therapy intervention
}

\begin{abstract}
Introduction: Stroke (CVA) can generate motor, sensory and cognitive development deficits, affecting the individual's performance in daily activities. Changes in any cognitive area can affect the individual's occupational engagement. Objective: To evaluate the cognitive and functional capacity in patients suffering from stroke, showing the importance of cognitive assessment for occupational therapy intervention. Method: A comparative study with cross-sectional sampling of 44 subjects aged 30-80 years, both sexes. The subjects were divided in three groups: Adult: 11 individuals affected by stroke, 30-59 years old; Elderly: 10 individuals affected by stroke, 60-80 years old; Control: 23 normal subjects, 30-80 years old. Tests applied: MMSE, Clock Test, Test of tracks A and B, and functional capacity (BOMFAQ). Results: Cognitive changes were identified in the Adult and Elderly groups. The Adult group showed poorer performance on the Clock test (visuospatial and executive functions) compared with the Control group. The Adult and Elderly groups presented worse performance in the Track A test (attention) compared with the Control group. In the Track B test (visual attention, graphomotor skills, and mental flexibility), applied with absolute numbers, no significant differences were observed between the Adult and Elderly groups and the Control group, but cognitive impairment was perceived when the test was applied with categories. The Adult group showed higher prevalence of moderate/severe impairment in the carrying out of daily activities. Conclusion: As a rule, individuals suffering from stroke, in addition to having impaired functional capacity, present cognitive impairments that can negatively impact the performance of daily tasks, whether they are occupational, leisure or self-care activities. Accordingly, we observed the need to evaluate cognitive rehabilitation for better targeting and quality of life improvement.
\end{abstract}

Keywords: Stroke, Cognition, Functionality, Occupational Therapy.

\section{Introdução}

Acidente vascular encefálico (AVE) tem grande potencial para gerar déficits no funcionamento motor, sensorial e cognitivo, com impacto no cotidiano e no desempenho do indivíduo frente às atividades $\mathrm{da}$ vida diária e afeta a avaliação subjetiva que ele faz da sua vida. À medida que as pessoas envelhecem, a capacidade de recuperarem-se de eventos negativos $\mathrm{da}$ vida adquire maior importância (KANH; JUSTER, 2002), ao mesmo tempo em que aumentam os riscos de perdas biológicas, cognitivas, motivacionais e sociais (FORTES; NERI, 2004).

O Brasil vem passando por um processo de inversão das curvas de mortalidade na qual ocorre um aumento por doenças crônicas não transmissíveis, principalmente o AVE. Segundo Bocchi e Angelo (2005), essas enfermidades, quando não são fatais, levam com frequência à dependência parcial ou total do indivíduo, com graves repercussóes para ele, sua família e a sociedade.

Segundo dados da Secretaria de Atenção à Saúde do Ministério da Saúde - SAS/MS, a ocorrência de internaçóes hospitalares por AVE na população de 30 a 59 anos foi de $\leq 4,7$ por 10 mil habitantes no ano de 2011 e de $\leq 5,2$ por 10 mil habitantes no ano de 2010 (BRASIL, 2011).
Apesar do vasto conhecimento biomédico já consolidado sobre o AVE, segundo Mackenzie e Chang (2002), as informaçôes acerca das consequências cognitivas, emocionais e comportamentais da doença ainda são controversas e escassas. De acordo com Doyle (2002), muitos estudos indicam mudanças na qualidade de vida das vítimas, mesmo entre aqueles moderadamente afetados.

Contudo, o paciente com AVE pode ter problemas com atividades cotidianas simples, como caminhar, vestir, comer e usar o banheiro. Problemas de movimentação podem resultar de dano à parte do cérebro que controla o equilíbrio e a coordenaçáo e ainda podem ocorrer problemas com raciocínio, atenção, aprendizado, julgamento e memória (FONTES, 2009).

Segundo Friedrich e Preiss (2006), à medida que o ser humano vai se desenvolvendo, os processos de aprendizagem modelam o cérebro, dissolvendo conexôes pouco utilizadas ou fortalecendo as ativas de uso frequente. O desenvolvimento da capacidade cognitiva e do cérebro estâo vinculados um ao outro de forma indissociável.

De acordo com Oliveira, Goretti e Pereira (2006), cognição é o termo empregado para descrever toda a esfera do funcionamento mental. Esse domínio implica a habilidade de sentir, pensar, perceber, lembrar, raciocinar, formar estruturas complexas de 
pensamento e a capacidade de produzir respostas às solicitaçóes e estímulos externos. Fatores educacionais, de saúde e de personalidade, bem como do nível intelectual global e capacidades mentais específicas do indivíduo podem contribuir para o declínio gradual das funçôes cognitivas, principalmente na senescência.

Com base no estudo de Freitas et al. (2006), uma disfunção cognitiva pode afetar a capacidade funcional em todas as esferas da vida diária: social e interpessoal, trabalho, lazer e atividades de vida diária.

Diante de situações sociais, as disfunções cognitivas podem interferir na habilidade de o indivíduo entender e integrar precisamente todas as partes de uma situação. Além disso, um paciente cognitivamente comprometido pode ter dificuldades em manter uma recordação de eventos anteriores e associar informaçóes relacionadas entre si.

Nesse sentido, a avaliação pode contribuir para o aumento da precisão diagnóstica, para a diminuiçáo dos internamentos hospitalares, do uso de medicamentos e para a redução dos gastos sanitários, constituindo-se, assim, no mais importante instrumento de planejamento das açóes de saúde para a população, de acordo com Kawamoto, Yoshida e Oka (2004).

Este trabalho se propôs avaliar a capacidade cognitiva e funcional em pacientes que sofreram AVE mostrando a importância da avaliaçáo cognitiva para a intervenção do terapeuta ocupacional.

\section{Método}

A pesquisa foi aprovada pelo Comitê de Ética em Pesquisa da Universidade Estadual de Ciências da Saúde de Alagoas - UNCISAL (1449/2011).

Trata-se de um estudo comparativo de delineamento transversal com amostra de 44 indivíduos na faixa etária de 30 a 80 anos, de ambos os sexos, distribuídos em três grupos: grupo Adulto, formado por 11 pacientes entre 30 e 59 anos, acometidos por AVE; grupo Idoso, formado por 10 pacientes entre 60 e 80 anos, acometidos por AVE; e grupo Controle, formando por 23 indivíduos sem episódio de AVC e com idade entre 30 a 80 anos. Os participantes dos três grupos responderam a um questionário sociodemográfico e foram submetidos a quatro testes para avaliação da capacidade cognitiva e um teste para avaliaçáo da capacidade funcional; os instrumentos foram aplicados de modo individual pelos pesquisadores.
Os sujeitos da pesquisa com sequelas de AVE foram recrutados nos Ambulatórios de Terapia Ocupacional e Fisioterapia da Universidade Estadual de Ciências da Saúde de Alagoas. Os indivíduos do grupo Controle foram recrutados de forma aleatória entre parentes e conhecidos dos pacientes. Os sujeitos da pesquisa foram avaliados no período de março a agosto de 2011.

Após a explanação dos procedimentos que seriam realizados durante a pesquisa e a assinatura do termo de consentimento livre e esclarecido, os participantes foram inquiridos sobre uso de medicamentos que poderiam alterar o comportamento, sobre uso de drogas psicotrópicas, bem como sobre alterações psiquiátricas ou neurológicas e sensoriais (dificuldades auditivas e/ou visuais e de fala) e alfabetização.

A capacidade cognitiva foi avaliada através dos seguintes testes: 1. Mini-Exame do Estado Mental (MEEM) (FOLSTEIN; FOLSTEIN; McHUGH, 1975), que avaliou a cognição global, envolvendo duas categorias de resposta, verbal e não verbal, e foi composto por 30 questóes categóricas, no qual adotamos a seguinte pontuação: 30 a 26 pontos: funçấo cognitiva preservada; 26 a 24 pontos: alteração não sugestiva de déficit; e 23 pontos ou menos: sugestivo de déficit cognitivo. 2. teste do relógio (SUNDERLAND et al., 1989), que avaliou as habilidades relacionadas a memória, habilidade visuoespacial e construtiva e funçôes executivas. 3 . O teste das trilhas A e B (STRAUSS; SHERMAN; SPREEN, 2006), que avaliou a atenção visual, a habilidade grafomotora e de sequência e a flexibilidade mental. Para avaliar a capacidade funcional utilizou-se a escala Functional Brazilian Older American Resources and Services Multidimensional Functional Assessment Questionnaire (Bomfaq), O Bomfaq avalia a dificuldade referida na realização de 15 atividades cotidianas, sendo oito relacionadas a atividades de vida diária (AVD): deitar/levantar da cama, comer, pentear o cabelo, andar no plano, tomar banho, vestir-se, ir ao banheiro em tempo e subir um lance de escada; e sete relacionadas às atividades instrumentais de vida diária (AIVD): medicar-se na hora, andar perto de casa, fazer compras, preparar refeiçôes, cortar unhas dos pés, sair de condução e fazer limpeza de casa. Considerou-se para cada um dos itens investigados dois tipos de respostas possíveis: sem dificuldade e com dificuldade (com ajuda e sem ajuda). Depois foram construídas categorias de acordo com o número de AVD/AIVD comprometidas. Foi utilizada análise descritiva. Aos dados provenientes dos questionários MEEM, BOMFAQ e teste do relógio foi aplicado o teste ANOVA. Nos casos em que ocorreu significância foi utilizado o pós-teste 
de Tukey, para demonstrar quais grupos diferiram entre si. Para análise dos testes de trilha A e B utilizou-se o teste do Qui-Quadrado, sendo o nível de significância adotado de $\mathrm{p}<0,05$.

\section{Resultados}

A amostra do estudo foi composta por 44 indivíduos, sendo $21 \mathrm{com}$ sequelas de AVE e 23 sem AVE formando o grupo Controle. A maioria era do sexo feminino $(56,8 \%)$, a média de idade foi de 59,43 $\pm 11,38$ anos, sendo a mínima de 30 anos e a máxima de 80 anos. A maioria $(88,64 \%)$ possuía entre 4 a 8 anos de escolaridade (Tabela 1).

Em relação às características clínicas, a doença crônica mais prevalente foi a HAS $(56,8 \%)$, seguida por dislipidemias $(25 \%)$ e reumatismo $(25 \%)$, conforme Tabela 2.

Em relação às queixas de memória, $56,8 \%$ as manifestaram na amostra total, distribuídos entre os grupos: Adulto (54,5\%): Idoso (60\%) e grupo Controle (65,2\%), conforme Tabela 3.

Os resultados dos testes cognitivos e funcional distribuídos na Tabela 4 mostram que não houve diferença significante no MEEM entre os três grupos, quando a comparação foi realizada utilizando-se os valores absolutos do teste. Entretanto, quando os indivíduos foram comparados de forma categórica, utilizando-se o ponto de corte $(\leq 24)$, os pacientes dos grupos Adulto e Idoso foram categorizados como apresentando alteração cognitiva.

$\mathrm{Na}$ análise do teste do relógio houve diferença significante no grupo Adulto, revelando alteração cognitiva em relação a memória, habilidade

Tabela 1. Características sociodemográficas da amostra.

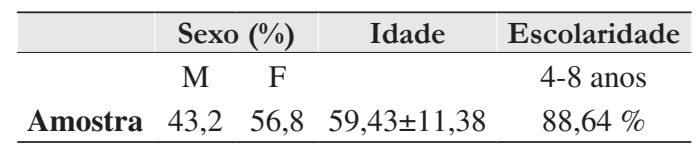

Tabela 2. Prevalência das doenças crônicas na amostra dos grupos.

\begin{tabular}{lc}
\hline & $\%$ \\
\hline Hipertensão Arterial Sistêmica (HAS) & 56,8 \\
Reumatismo & 25 \\
Dislipidemias (colesterol alto) & 25 \\
Diabetes Mellitus & 18,2 \\
Doenças cardíacas & 11,4 \\
Problemas respiratórios & 11,4 \\
Disfunção tireodiana & 6,8 \\
\hline
\end{tabular}

visuoespacial e construtiva e função executiva, quando comparado com os grupos Idoso e Controle.

Em relação à análise do teste trilha $\mathrm{A}$, houve diferença significante nos grupos Adulto e Idoso em relação ao grupo Controle. Os grupos Adulto e Idoso apresentaram alteração cognitiva (atenção visual, habilidade grafomotora, de sequência e flexibilidade mental). Por outro lado percebemos que não houve diferença significante nos grupos Adulto e Idoso em relação ao teste trilha $\mathrm{B}$, quando utilizaram-se os números absolutos do teste, em relação ao grupo Controle. Entretanto, quando os grupos Adulto e Idoso foram analisados levando-se em consideração as categorias da escala, observou-se haver alteraçáo cognitiva (atenção visual, habilidade grafomotora, de sequência e flexibilidade mental).

$\mathrm{Na}$ análise da capacidade funcional (BOMFAQ), em relação ao desempenho nas AVD/AIVD, o grupo Idoso apresentou maior prevalência no comprometimento moderado e o grupo Adulto maior comprometimento funcional grave (Figura 1).

\section{Discussão}

O estudo mostra que após acidente vascular encefálico foram identificadas alteraçôes cognitivas que afetam a independência nas atividades cotidianas nos grupos Adulto e Idoso. Assim como no estudo de Ramos, Simoes e Albert (2001), que demonstraram que o comprometimento cognitivo está associado a dependência nas atividades da vida diária e fortemente relacionado com mortalidade.

Alteraçôes cognitivas podem ocorrer como sinais de doenças neurológicas e até mesmo no envelhecimento normal e muitas vezes não são relatadas ou valorizadas pelos pacientes e familiares e até mesmo pelos profissionais. Nosso estudo mostra um grande número de pacientes acometidos de AVE nos grupos Adulto e Idoso que apresentaram déficit cognitivo $(\leq 24$ no MEEM) cujas queixas de dificuldade na memória não tinham sido ainda investigadas ou mesmo valorizadas durante o tratamento. Esse resultado é condizente com o estudo de Vitiello et al.

Tabela 3. Queixas de memória na amostra por grupos.

\begin{tabular}{lcc}
\hline \multicolumn{1}{c}{ Queixa de memória } & \multicolumn{2}{c}{$\%$} \\
& SIM & Não \\
Grupo Adulto (AVE) & 54,5 & 45,5 \\
Grupo Idoso (AVE) & 60,0 & 40,0 \\
Grupo Controle & 65,2 & 34,8 \\
Amostra total & 56,8 & 43,2 \\
\hline
\end{tabular}


Tabela 4. Distribuição dos resultados dos testes da avaliação da capacidade cognitiva e funcional nos grupos.

\begin{tabular}{lccccc}
\hline & MEEM & Relógio & Trilha A (\%) & Trilha B (\%) & BOMFAQ \\
\hline Grupo Adulto (AVE) & $21,2 \pm 7,65$ & $5,5 \pm 3,29^{*}$ & $54,5^{*}$ & 45,45 & $3,2 \pm 1,25^{*}$ \\
Grupo Idoso (AVE) & $22,5 \pm 6,46$ & $6,9 \pm 3,14$ & $60,0^{*}$ & 10 & $2,8 \pm 0,91$ \\
Grupo Controle & $26,4 \pm 2,65$ & $9,3 \pm 1,63$ & 95,65 & 43,47 & $1,34 \pm 0,57$ \\
\hline
\end{tabular}

${ }^{*} \mathrm{P}<0,05$.

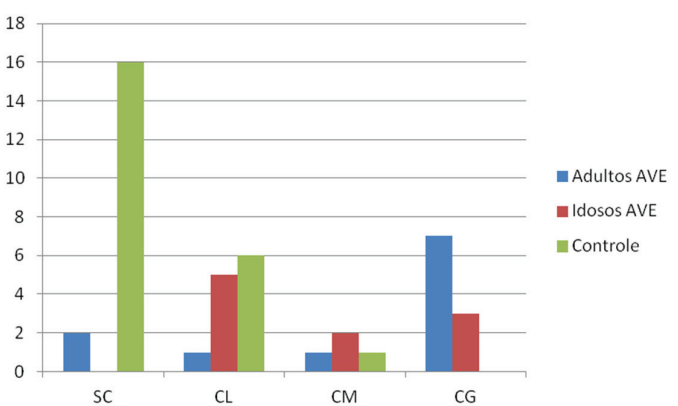

Figura 1. Escala de avaliação multidimensional do estado funcional (BOMFAQ) (SC, sem comprometimento; CL, comprometimento leve; CM, comprometimento moderado; CG, comprometimento grave).

(2007), que ressalta a necessidade de realizar a avaliação dessas funçôes, principalmente em pacientes neurológicos.

Outro estudo (SCALZO et al., 2010) mostrou que 44 pacientes pós-AVE foram avaliados e que apenas 26 obtiveram escores acima de 18 pontos no MEEM. O escore médio obtido foi $18,7( \pm 5,9)$, sendo esse resultado menor que o encontrado no nosso.

Nosso estudo revelou que a maioria dos indivíduos, além dos acometidos pelo AVE, também aqueles sem acometimento (grupo Controle), relataram déficit de memória, mostrando ser necessário a realização de uma avaliação cognitiva em pessoas com idade de 50 anos ou mais para que sejam trabalhadas a prevenção ou mesmo estratégias para melhorar o seu desempenho nas atividades cotidianas.

Nossos achados, quando comparados ao estudo de Quadros Junior et al. (2008), diferem do perfil do estado cognitivo geral em idosos. $\mathrm{O}$ referido estudo relatou valores médios sugestivos de quadro degenerativo, quando analisados pelas notas sugeridas para a populaçáa brasileira. Ele enfatiza a sugestáo de que essas perdas devam-se ao avançado da idade, devido a perda de tecido cerebral, ou mesmo a baixa escolaridade encontrada nesses idosos.

A literatura tem revelado maior comprometimento cognitivo associado à idade em idosos, porém, em nossos achados, a idade não foi fator determinante, pois maior prevalência de déficit cognitivo revelou-se no grupo Adulto.

Estudo similar ao nosso (BENNETT et al., 2002) mostrou que a maior parte da população idosa náo apresenta declínio cognitivo, ou seja, apresenta trajetória evolutiva estável e benigna sem prejuízo das AVD, desde que as doenças crônicas estejam controladas.

Em relação ao comprometimento no desempenho das atividades cotidianas, encontramos maior prevalência nos grupos Adulto $(72,7 \%)$ e Idoso (50\%). Esse resultado mostra ainda que a idade também não foi um fator determinante no nível de comprometimento funcional do indivíduo.

Nosso estudo aponta maior prevalência em relação ao estudo de Baer e Smith (2001), que revelaram que cerca de $30 \%$ a $60 \%$ dos sujeitos pós-AVE ficaram em situação de dependência. $\mathrm{O}$ estudo de Nunes, Pereira e Silva (2005) encontrou que 40\% dos pacientes pós-AVE ficaram dependentes de terceiros para a realizaçáo de suas atividades diárias.

O grupo Adulto apresentou maior déficit na cognição e no comprometimento funcional na realização das AVD, quando comparado aos outros grupos. Para esclarecermos essa situação recorremos ao estudo de Kanh e Juster (2002), que sugerem que as degeneraçôes normais de pequena monta decorrentes do envelhecimento permitiriam, através da neuroplasticidade, que o cérebro do idoso resista de forma mais adequada aos danos provocados pelo AVE, havendo claras indicaçôes de que o nível de saúde física e mental e a funcionalidade refletem-se na capacidade para o desempenho de atividades básicas e instrumentais da vida diária.

A Organização Mundial de Saúde (OMS) (1997) destaca que a capacidade funcional e a independência são fatores preponderantes para o diagnóstico de saúde física e mental na população idosa.

Segundo Doyle (2002), o indivíduo acometido por um AVE apresenta sérias limitaçôes em múltiplos domínios do funcionamento físico, cognitivo e social que prejudicam as atividades cotidianas; com isso, podemos refletir que a realização adequada de uma tarefa do cotidiano envolve a participaçáo das funções cognitivas, motoras e psicológicas. 
Gonçalves et al. (2010) referem que a detecção do grau de dependência nos diferentes contextos possibilita selecionar intervençôes adequadas, aplicadas tanto individualmente quanto em grupo, com vistas à melhoria da capacidade funcional no desempenho das atividades cotidianas. Ávila (2003) reforça que a diminuição dos déficits que ocasionam afastamento e isolamento social, dependência e discriminação também contribuem para o bem-estar. Além disso, de acordo com Nishida, Amorim e Inoue (2004), os estímulos devem buscar otimizar a reorganização cerebral a partir do treino durante as tarefas, através dos estímulos terapêuticos e reorganização do ambiente sociofamiliar.

A Terapia Ocupacional possibilita ao indivíduo "ser reconhecido e se reconhecer por seus fazeres", permite conhecer a história de vida dos sujeitos, visando a conquista da independência e a organização de um cotidiano potencializado e vivificado, no sentido da construção do bem-estar pessoal e do empreendimento de lutas para tudo o que implica a construção da qualidade de vida (DE CARLO; BARTALOTTI, 2001).

Dessa maneira, de acordo com os resultados obtidos em nosso estudo, percebemos a importância da avaliação cognitiva e consequentemente da reabilitação, que implica em maximizar funçóes cognitivas por meio do bem-estar psicológico, da habilidade em AVD e do relacionamento social. Diante dessas consideraçóes, o tratamento da Terapia Ocupacional pode ajudar o indivíduo acometido de AVE a melhorar seu desempenho no dia a dia, permitindo que ele seja mais independente e que possa receber menor ajuda de terceiros.

Acreditamos que a avaliação da capacidade cognitiva poderá orientar intervençôes específicas, sendo o acompanhamento cognitivo fundamental na promoção de uma vida mais ativa.

Contudo, o terapeuta ocupacional, entre outras ações, deve estar preocupado em auxiliar o indivíduo a reiniciar ou reconstruir sua história ocupacional e ser ator das atividades importantes em sua vida, mesmo sendo portador de doenças ou incapacidades.

Sendo assim, verificamos através desse estudo a importância de se conhecer o indivíduo de forma ampla, através da avaliação funcional e cognitiva, para que o tratamento terapêutico ocupacional possa alcançar seus objetivos.

\section{Conclusão}

Essa pesquisa revela que indivíduos acometidos de acidente vascular encefálico apresentam alteraçôes cognitivas importantes, além do comprometimento funcional no que tange à realização das tarefas cotidianas, sejam elas ocupacionais, de lazer ou mesmo as básicas de autocuidado e as de relação com outros indivíduos (família e ambiente).

As alteraçôes cognitivas envolvem quase todas as áreas da cognição (orientação espaço-temporal, memória, atenção, linguagem e funçôes executivas), havendo intrínseca relação com o desempenho nas atividades cotidianas.

O estudo revelou a necessidade de se avaliar o indivíduo de forma ampla, verificando as dificuldades/ déficits e também as habilidades remanescentes, ou seja, de se avaliar a capacidade cognitiva para traçar medidas que o ajudem a aumentar seu bem-estar e grau de independência.

Nesse sentido, considerando-se os déficits cognitivos e funcionais encontrados nos indivíduos com sequelas de AVE, verificamos a importância da avaliação e reabilitação cognitiva para que esses indivíduos aprendam a desenvolver estratégias que elevem sua capacidade no desempenho das atividades cotidianas e fortaleçam sua independência e autonomia, melhorando sua qualidade de vida.

\section{Referências}

ÁVILA, R. Resultados da reabilitação neuropsicológica em paciente com doença de Alzheimer leve. Revista de Psiquiatria Clínica, São Paulo, v. 30, n. 4, p. 139-146, 2003.

BAER, G.; SMITH, M. The recovery of walking ability and subclassification of stroke. Physiotherapy Research International, Hoboken, v. 6, n. 3, p. 135-44, 2001. http://dx.doi.org/10.1002/pri.222

BENNETT, D. A. et al. Natural history of mild cognitive impairment in older persons. Neurology, Minneapolis, v. 59, n. 2, p. 198-205, 2002. http://dx.doi.org/10.1212/ WNL.59.2.198

BOCCHI, S. C. M.; ANGELO, M. Interação cuidador família-pessoa com AVC: autonomia compartilhada. Ciência \& Saúde Coletiva, Rio de Janeiro, v. 10, n. 3, p. 729-738, 2005.

BRASIL. Ministério da Saúde. Secretaria-Executiva. Departamento de Apoio à Gestão Descentralizada. Orientações acerca dos indicadores de monitoramento avaliação do pacto pela saúde, nos componentes pela vida e de gestão para o biênio 2010-2011. Brasília: Ministério da Saúde, 2011. Disponível em: <http://portalweb04. saude.gov.br/sispacto/Instrutivo_Indicadores_2011.pdf>. Acesso em: 03 nov. 2011.

DE CARLO, M. M. R. P.; BARTALOTTI, C. C. Terapia ocupacional no Brasil: fundamentos e perspectivas. 2. ed. Plexus: São Paulo, 2001. PMCid:PMC101159.

DOYLE, P. J. Measuring health outcomes in stroke survivors. Archives of Physical Medicine and Rehabilitation, Philadelphia, v. 83, n. 12, p. S39-43, 2002. Supplement 2. 
FOLSTEIN, M. F.; FOLSTEIN, S. E.; McHUGH, P. R. "Mini-mental state": a practical method for grading the cognitive state of patients for the clinician. Journal of Psychiatric Research, Oxford, v. 12, n. 3, p. 189-198, 1975. http://dx.doi.org/10.1016/0022-3956(75)90026-6

FONTES, H. A. F. National Institute of Neurological Disorders and Stroke (NINDS). [s.l.: s.n.], 2009.

FORTES, A. C. G.; NÉRI, A. L. Eventos de vida e envelhecimento humano. In: NÉRI, A. L.; YASSUDA, M. S.; CACHIONI, M. (Org.). Velhice bem-sucedida: aspectos afetivos e cognitivos. Campinas: Papirus, 2004. p. 51-70.

FREITAS, E. V. et al. Tratado de geriatria e gerontologia. 2. ed. Rio de Janeiro: Guanabara Koogan, 2006.

FRIEDRICH, G; PREISS, G. Educar com a cabeça: Ao aprendermos, nossas conexōes cerebrais se modificam. Com o apoio da neurodidática, neurocientistas poderão ajudar professores e pedagogos a desenvolver novas estratégias de ensino e aprendizado. Viver Mente \& Cérebro, Sáo Paulo, v. 157, n. 1, p. 51-57, 2006.

GONÇALVES, L. H. T. et al. O idoso institucionalizado: avaliação da capacidade funcional e aptidão física. Cadernos de Saúde Pública, Rio de Janeiro, v. 26, n. 9, p. 1738-1746, set. 2010.

KANH, R. L.; JUSTER, F. T. Well-being: concepts and measures. Journal of Social Issues, Malden, v. 58, n. 4, p. 627-644, 2002. http://dx.doi. org/10.1111/1540-4560.00281

KAWAMOTO, R.; YOSHIDA, O.; OKA, Y. Factors related to functional capacity in communitydwelling elderly. Geriatrics Gerontology International, Tokyo, v. 4, n. 2, p. 105-110, 2004. http://dx.doi. org/10.1111/j.1447-0594.2004.00130.x

MACKENZIE, A. E.; CHANG, A. M. Predictors of quality of life following stroke. Disability and Rehabilitation, London, v. 24, n. 5, p. 259-265, 2002. http://dx.doi.org/10.1080/09638380110081805

NISHIDA, A. P.; AMORIM, M.; INOUE, M. Z. M. Índice de Barthel e do estado funcional de pacientes pós acidente vascular cerebral em programa de fisioterapia. Salusvita, Bauru, v. 23, n. 3, p. 467-77, 2004.
NUNES, S.; PEREIRA, C.; SILVA, M. G. Evolução funcional de utentes após AVC nos primeiros seis meses após a lesão. EssFisiOnline, Setúbal, v. 1, n. 3, p. 3-20, 2005.

OLIVEIRA, D. L. C.; GORETTI, L. C.; PEREIRA, L. S. M. O desempenho de idosos institucionalizados com alteraçóes cognitivas em atividades de vida diária e mobilidade: estudo piloto. Revista Brasileira de Fisioterapia, São Carlos, v. 10, n. 1, p. 91-96, 2006.

ORGANIZAÇÃO MUNDIAL DE SAÚDE - OMS. Centro Colaborador da OMS para a Classificação de Doenças em Português. CID-10 - Classificaçáo Internacional de Doenças. 4. ed. São Paulo: Edusp, 1997. v. 1. p. 9-29.

QUADROS JUNIOR, A. C. et al. Estudo do nível de atividade física, independência funcional e estado cognitivo de idosos institucionalizados: análise por gênero. Brazilian Journal of Biomotricity, Rio Claro, v. 2, n. 1, p. 39-50, 2008.

RAMOS, L. R.; SIMOES, E. J.; ALBERT, M. $S$. Dependence in activities of daily living and cognitive impairment strongly predicted mortality in older urban residents in Brazil: a 2-year follow-up. Journal of the American Geriatrics Society, New York, v. 49, n. 9, p. 1168-1175, 2001. http://dx.doi. org/10.1046/j.1532-5415.2001.49233.x

SCALZO, P. L. et al. Qualidade de vida em pacientes com Acidente Vascular Cerebral: clínica de fisioterapia Puc Minas Betim. Revista Neurociências, Sáo Paulo, v. 18, n. 2, p. 139-144, 2010.

STRAUSS, E.; SHERMAN, E. M. S.; SPREEN, O. A compedium of neuropshychological test: administration, norms, and commentary. Oxford: Oxford University Press, 2006.

SUNDERLAND, T. et al. Clock drawing in Alzheimer's disease: a novel measure of dementia severity. Journal of the American Geriatrics Society, New York, v. 37, n. 8, p. 725-729, 1989.

VITIELLO, A. P. P. et al. Avaliação cognitiva breve de pacientes atendidos em ambulatórios de neurologia geral. Arquivos de Neuro-psiquiatria, São Paulo, v. 65, n. 2-A, p. 299-303, 2007. http://dx.doi.org/10.1590/ S0004-282X2007000200021

\section{Contribuição dos Autores}

Andressa de Oliveira Ferro: Trabalhou na concepção do estudo e seu delineamento, na consecução da metodologia, na análise e interpretação dos resultados e na redação do manuscrito. Ana Elizabeth dos Santos Lins: Trabalhou na metodologia, na análise e interpretação dos resultados, na redação e revisão crítica do manuscrito. Euclides Mauricio Trindade Filho: Trabalhou na metodologia, na análise estatística dos resultados. 